\title{
ON THE DIVISION OF DISTRIBUTIONS BY ANALYTIC FUNCTIONS IN LOCALLY CONVEX SPACES \\ BY \\ DENIS CHANSOLME
}

\begin{abstract}
Although the division of distributions by real polynomials and real analytic functions (which are nonzero) is always possible in finite dimensional spaces (from classical results of Hörmander and Lojasiewicz respectively), we show that this is not always possible in infinite dimensional locally convex spaces. In particular, we characterize those locally convex spaces where division is always possible.
\end{abstract}

I. Introduction. Let $S$ be a distribution and $g$ a $C^{\infty}$ function on an open subset $\Omega$ in a locally convex space. We say that $g$ divides $S$ if there exists a distribution $T$ on $\Omega$ so that $g T=S$.

This problem was first studied by L. Schwartz [8] who proved that the division by a nonzero holomorphic function is always possible on a connected open subset of $\mathbf{C}^{n}$. Later, L. Hörmander [6] and S. Lojasiewicz [7] respectively solved the division by real polynomials and analytic functions in finite dimensional spaces. J. F. Colombeau, R. Gay and B. Perrot [4] extended Schwartz's result to complex locally convex spaces.

These works established that if $g$ does not vanish on any open subset of $\Omega$, the division is always possible. Our purpose is to answer a natural question in Colombeau, Gay and Perrot [4] concerning division by real polynomials and real analytic functions in infinite dimensional locally convex spaces.

We prove division is always possible by finite type real polynomials and finite type real analytic functions which are nonzero (defined later). But the division by general polynomials is not possible in general. We characterize the spaces where division is always possible and will see that they form a rather limited class; in particular, the unique infinite dimensional Fréchet space where division by nonzero real polynomials or real analytic functions is always possible is $\mathbf{R}^{\mathbf{N}}$, and the unique Silva space is $\mathbf{R}^{(\mathbf{N})}$.

II. Notation. They are classical. We denote by $E$ a Hausdorff real locally convex space and $\Omega$ an open subset of $E$. A mapping $f: \Omega \rightarrow \mathbf{R}$ is called a $C^{\infty}$ function if, for every convex balanced bounded subset $B$ of $E$, the restriction of $f$ to $\Omega \cap E_{B}$ (where $E_{B}$ denotes, as usual, the vector space spanned by $B$ and normed with the gauge $\rho_{B}$ of $B$ ) is $C^{\infty}$ in the usual Fréchet sense of calculus in normed spaces. (This is the

Received by the editors May 19, 1981 and, in revised form, October 27, 1981.

AMS (MOS) subject classifications (1970). Primary 46F25; Secondary 46G05, 46G20, 46B99.

Key words and phrases. Infinite dimensional distributions, division by analytic functions. 
definition of the Silva $C^{\infty}$ function on $\Omega$-see Colombeau [2] for details on this concept.)

We denote by $\mathcal{E}(\Omega)$ the vector space of the $C^{\infty}$ functions on $\Omega$. We consider on $\mathcal{E}(\Omega)$ the topology of the uniform convergence of functions and all their derivatives on the strictly compact subsets of $\Omega$ (i.e. compact in some $\Omega \cap E_{B}$ ). A basis of neighborhoods of zero in $\mathcal{E}(\Omega)$ is made of sets $V(K, L, \varepsilon, \alpha)=\{f \in \mathcal{E}(\Omega)$ such that $\sup _{x \in K ; h_{j} \in L}\left|f^{(i)}(x) h_{1} \cdots h_{i}\right| \leqslant \varepsilon$ if $\left.0 \leqslant i \leqslant \alpha\right\}$ where $K$ is a strictly compact subset of $\Omega, L$ a bounded subset of $E, \varepsilon>0$ and $\alpha \in \mathbf{N}$. We call here "distribution on $\Omega$ " any element of the dual space $\mathcal{E}^{\prime}(\Omega)$ of $\mathcal{E}(\Omega)$.

The function $g$ is called analytic on $\Omega$ if for each balanced convex bounded subset $B$ of $E$, the restriction of $f$ to $\Omega \cap E_{B}$ is locally the sum of a normally convergent series of $E_{B}$-continuous polynomials (see Colombeau [3]).

An analytic function $g$ on $\Omega$ is said to be of finite type if there exist two subspaces $E_{1}$ and $E_{2}$ of $E$ such that $E$ can be decomposed as the topological direct sum $E=E_{1} \oplus E_{2}$ with $\operatorname{dim} E_{1}<\infty$, and if for all $x \in \Omega, x=x_{1}+x_{2}\left(x_{1} \in E_{1}, x_{2} \in\right.$ $\left.E_{2}\right)$, we have $g\left(x_{1}\right)=g(x)$.

We notice that if $g$ is a polynomial on $E$, this definition coincides with the usual one for finite type continuous polynomials. We denote by $p_{1}$ and $p_{2}$ the two projections:

$$
P_{1}: \begin{aligned}
& E_{1} \oplus E_{2} \rightarrow E_{1} \\
& x_{1}+x_{2} \mapsto x_{1}
\end{aligned} \text { and } p_{2}: \begin{gathered}
E_{1} \oplus E_{2} \rightarrow E_{2} . \\
x_{1}+x_{2} \mapsto x_{2}
\end{gathered} .
$$

If $V$ is a convex balanced neighborhood of zero, we denote by $\rho_{V}$ or $\|\cdot\|_{V}$ the gauge of $V, E_{V}=E / \rho_{V}^{-1}(0)$ and $s_{V}: E \rightarrow E / \rho_{V}^{-1}(0)$ the canonical surjection map.

The notation $\stackrel{\circ}{V} \stackrel{\circ}{B}, V(K, L, \varepsilon, \alpha)^{0}$ will be used for the polars of $V, B, V(K, L, \varepsilon, \alpha)$ respectively.

\section{Division by finite type analytic functions.}

THEOREM 1. Let $E$ be a Hausdorff real locally convex space, $\Omega$ an open subset of $E$ and $g$ a locally finite type analytic function on $\Omega$ which does not vanish identically on any open subset. Then, for every $S \in \mathcal{E}^{\prime}(\Omega)$ there exists a $T \in \mathcal{E}^{\prime}(\Omega)$ such that $S=g T$.

Proof. Let us consider the map

$$
u: \mathcal{E}(\Omega) \rightarrow \mathcal{E}(\Omega), \quad f \mapsto g f .
$$

We remark that this mapping is injective because $g$ does not vanish on any open subset of $\Omega$. Therefore, it suffices to prove that $u$ is open. Indeed, if $S \in \mathcal{E}^{\prime}(\Omega)$ the linear form

$$
g \mathcal{E}(\Omega) \rightarrow \mathcal{E}(\Omega) \rightarrow \mathbf{R}, \quad g f \mapsto f \mapsto\langle S, f\rangle
$$

would be continuous. Thus from the Hahn-Banach theorem it would be extended to $\mathcal{E}(\Omega)$ in a $T \in \mathcal{E}^{\prime}(\Omega)$, and then $S=g T$.

For every $x_{0} \in \Omega$, there exist two subspaces $E_{1}$ and $E_{2}$ of $E$ such that $E=E_{1} \oplus E_{2}$ topologically, with $\operatorname{dim} E_{1}<\infty$, and two convex balanced open neighborhoods of zero, $V_{x_{0}}$ and $W_{x_{0}}$ respectively in $E_{1}$ and $E_{2}$, with $x_{0}+V_{x_{0}}+W_{x_{0}} \subset \Omega$, such that if 
we set $\Omega_{1}=p_{1}\left(x_{0}\right)+V_{x_{0}}$ and $\Omega_{2}=p_{2}\left(x_{0}\right)+W_{x_{0}}$ we have $g\left(\xi+y_{1}\right)=g\left(\xi+y_{2}\right)$ for all $\xi \in \Omega_{1}$ and $y_{1}, y_{2} \in \Omega_{2}$. Let $K_{1}$ and $K_{2}$ be two strictly compact subsets of $\Omega_{1}$ and $\Omega_{2}$ respectively and $B$ a convex balanced bounded subset of $E_{1}$. For every $x \in \Omega$, we have

$$
\begin{aligned}
& \sup _{\substack{h_{i} \in B \\
i=1 \cdots n}}\left|f^{(n)}(x) h_{1} \cdots h_{n}\right| \\
& \leqslant 2^{n} \sup _{\substack{h_{i} \in B \\
i=1 \cdots n \\
k=0 \cdots n}}\left|f^{(n)}(x) p_{1}\left(h_{1}\right) \cdots p_{1}\left(h_{k}\right) p_{2}\left(h_{k+1}\right) \cdots p_{2}\left(h_{n}\right)\right| .
\end{aligned}
$$

Let us fix $k \in\{0 \cdots n\}$ and $l_{k+1}, \ldots, l_{n} \in B$. If $x \in \Omega_{1}+\Omega_{2}$, we set $F(x)=$ $f^{(n-k)}(x) p_{2}\left(l_{k+1}\right) \cdots p_{2}\left(l_{n}\right)$. If $x=\xi+y \in K_{1}+K_{2}\left(\xi \in K_{1}, y \in K_{2}\right)$ we set

$$
F_{y}: \Omega_{1} \rightarrow \mathbf{R}, \quad t \mapsto F_{y}(t)=F(t+y) .
$$

Then we have

$$
\begin{aligned}
& \sup _{\substack{h_{i} \in B \\
i=1 \cdots k}}\left|F^{(k)}(x) p_{1}\left(h_{1}\right) \cdots p_{1}\left(h_{k}\right)\right|=\sup _{\substack{h_{i} \in B \\
i=1 \cdots k}}\left|F_{y}^{(k)}(\xi) p_{1}\left(h_{1}\right) \cdots p_{1}\left(h_{k}\right)\right| \\
& \leqslant \sup _{\substack{h_{i} \in p_{1}(B) \\
i=1 \cdots k \\
t \in K_{1}}}\left|F_{y}^{(k)}(t) h_{1} \cdots h_{k}\right|
\end{aligned}
$$

The function $F_{y}$ belongs to $\mathcal{E}\left(\Omega_{1}\right), K_{1}$ is a compact subset of $\Omega_{1}, \Omega_{1}$ is an open subset of $E_{1}, \operatorname{dim} E_{1}<\infty$ and $g$ does not vanish identically on any open subset of $\Omega_{1}$. Acccording to Lojasiewicz' result [7], there exist a compact subset $K_{1}^{\prime}$ of $\Omega_{1}$, a convex balanced bounded subset $B^{\prime}$ of $E_{1}, m_{k} \in \mathbf{N}$ and $C_{1}>0$ independent of $x$, such that

$$
\sup _{\substack{h_{i} \in p_{1}(B) \\ i=1 \cdots k \\ t \in K_{1}}}\left|F_{y}^{(k)}(t) h_{1} \cdots h_{k}\right| \leqslant C_{1} \sup _{\substack{h_{i}^{\prime} \in B^{\prime} \\ p<m_{k} \\ t \in K_{1}^{\prime}}}\left|\left(g F_{y}\right)^{(p)}(t) h_{1}^{\prime} \cdots h_{p}^{\prime}\right| .
$$

But $\left(g F_{y}\right)^{(p)}(t) h_{1}^{\prime} \cdots h_{p}^{\prime}=(g F)^{(p)}(t+y) h_{1}^{\prime} \cdots h_{p}^{\prime}$ since $h_{i}^{\prime} \in E_{1}$ and $(g F)(x)=$ $g(x) f^{(n-k)}(x) p_{2}\left(l_{k+1}\right) \cdots p_{2}\left(l_{n}\right)$. If we remark that $g^{(r)}(x) p_{2}\left(l_{1}^{\prime}\right) \cdots p_{2}\left(l_{r}^{\prime}\right)=0$ when $r \geqslant 1$ and $l_{1}^{\prime}, \ldots, l_{r}^{\prime} \in B$, applying the Leibnitz formula we have

$$
(g f)^{(n-k)}(x) p_{2}\left(l_{k+1}\right) \cdots p_{2}\left(l_{n}\right)=g(x) f^{(n-k)}(x) p_{2}\left(l_{k+1}\right) \cdots p_{2}\left(l_{n}\right) .
$$

Therefore, for $x \in K_{1}+K_{2}$

$$
\begin{aligned}
& \sup _{\substack{h_{i} \in B \\
i=1 \cdots k}}\left|f^{(n)}(x) p_{1}\left(h_{1}\right) \cdots p_{1}\left(h_{k}\right) p_{2}\left(l_{k+1}\right) \cdots p_{2}\left(l_{n}\right)\right| \\
& \leqslant C_{1} \sup _{\substack{h_{i}^{\prime} \in B^{\prime} \\
p \leqslant m \\
x \in K_{1}^{\prime}+K_{2}}}\left|(g f)^{(n-k+p)}(x) h_{1}^{\prime} \cdots h_{p}^{\prime} p_{2}\left(l_{k+1}\right) \cdots p_{2}\left(l_{n}\right)\right| .
\end{aligned}
$$


If $m=\sup _{k=0 \ldots n} m_{k}, B^{\prime \prime}=B^{\prime}+p_{2}(B)$ and $K^{\prime \prime}=K_{1}^{\prime}+K_{2}$, we obtain a first inequality

$$
\sup _{\substack{h_{i} \in B \\ x \in K_{1}+K_{2}}}\left|f^{(n)}(x) h_{1} \cdots h_{n}\right| \leqslant C_{1} \sup _{\substack{h_{i} \in B^{\prime \prime} \\ p \leqslant m \\ x \in K^{\prime \prime}}}\left|(g f)^{(n-k+p)}(x) h_{1} \cdots h_{n-k+p}\right| .
$$

Now let $K$ be a strictly compact subset of $\Omega$. In the same way, for every $x \in K$, we can define convex balanced open neighborhoods of zero: $V_{x}$ and $W_{x}$ (of some $E_{1}$ and $E_{2}$ which are dependent on $x$ ). We can find therefore a finite set $I$ such that $K \subset \cup_{i \in I} x_{i}+\frac{1}{2} V_{x_{i}}+\frac{1}{2} W_{x_{i}}$. If we set $K_{i}=K \cap\left(x_{i}+\frac{1}{2} \bar{V}_{x_{i}}+\frac{1}{2} \bar{W}_{x_{i}}\right)$, we have the following inclusions:

$$
K_{i} \subset p_{i 1}\left(K_{i}\right)+p_{i 2}\left(K_{i}\right) \subset\left(p_{i 1}\left(x_{i}\right)+V_{x_{i}}\right)+\left(p_{i 2}\left(x_{i}\right)+W_{x_{i}}\right) \subset \Omega
$$

with $p_{i 1}$ and $p_{i 2}$ the projections on $E_{i 1}$ and $E_{i 2}\left(E=E_{i 1} \oplus E_{i 2}\right.$ defined by $\left.x_{i}\right)$. The sets $p_{i 1}\left(K_{i}\right)$ and $p_{i 2}\left(K_{i}\right)$ being strictly compact in $E_{i 1}$ and $E_{i 2}$ respectively, we can then apply our previous result: if $\alpha \in \mathbf{N}$ there exist a strictly compact $K^{\prime}$ of $\Omega$, a bounded subset $B^{\prime}$ of $E, m^{\prime} \in \mathbf{N}, C>0$ such that, for every $\varepsilon>0, g f \in$ $V\left(K^{\prime}, B^{\prime}, \varepsilon / C, m^{\prime}\right)$ implies $f \in V(K, B, \varepsilon, \alpha)$. Therefore $u$ is open.

\section{Characterization of the spaces where division is always possible.}

THEOREM 2. Let $E$ be a Hausdorff real locally convex space, and $\Omega$ an open subset of $E$. The division by a nonzero continuous polynomial is always possible if and only if for every convex balanced neighborhood of zero $V$ and for every convex balanced bounded subset $B$ of $E$ we have $\operatorname{dim} s_{V}\left(E_{B}\right)<\infty$.

Proof. First part: Let us suppose there exist a convex balanced 0-neighborhood $V$ in $E$, and a convex balanced bounded subset $B$ of $E$ with $\operatorname{dim} s_{V}\left(E_{B}\right)=\infty$. We may assume $B \subset V$. By induction, there is a system $\left(e_{n}\right)_{n \in \mathrm{N}^{*}}$ with $e_{n} \in s_{V}(B)$, and $v_{n} \in\left(E_{V}, p_{V}\right)^{\prime}$ with $v_{n}\left(e_{m}\right)=0$ if and only if $n \neq m$. We may assume $\left\|v_{n}\right\|_{V}=1$ and $v_{n}\left(e_{n}\right)>0$. Let us set $a_{n}=n^{-1} v_{n}\left(e_{n}\right)$ and

$$
P_{V}: E_{V} \rightarrow \mathbf{R}, \quad x \mapsto \sum_{n \in \mathbf{N}^{*}} a_{n}^{n} v_{n}(x)^{2} .
$$

We have

$$
a_{n} \leqslant \frac{\left\|v_{n}\right\|_{V}\left\|e_{n}\right\|_{V}}{n} \leqslant \frac{1}{n} \text { and }\left|P_{V}(x)\right| \leqslant \sum_{n \in \mathrm{N}^{*}} n^{-n}\|x\|_{V}^{2}
$$

$P_{V}$ is thus a continuous polynomial on $E$. Therefore, $P=P_{V} \circ s_{V}$ is a continuous polynomial on $E$. Since $e_{n} \in s_{V}(B)$, there exists a $j_{n} \in B$ such that $s_{V}\left(j_{n}\right)=e_{n}$. Hence, we have $\left\|j_{n}\right\|_{B} \leqslant 1$ and $\left(n^{-1} j_{n}\right)_{n \in \mathrm{N}^{*}}$ converges to zero in $\left(E_{B}, \rho_{B}\right)$.

By translation, we may suppose that $0 \in \Omega$; so there is an $n_{0} \in \mathbf{N}^{*}$ such that $n^{-1} j_{n} \in \Omega$ if $n \geqslant n_{0}$. Let us define $S \in \mathcal{E}^{\prime}(\Omega)$ by

$$
\mathcal{E}(\Omega) \rightarrow \mathbf{R}, \quad f \mapsto\langle S, f\rangle=\sum_{n \geqslant n_{0}} n^{-2} f\left(n^{-1} j_{n}\right),
$$


and let us suppose there is a $T \in \mathcal{E}^{\prime}(\Omega)$ with $\langle T, P f\rangle=\langle S, f\rangle, \forall f \in \mathcal{E}(\Omega)$. Therefore there exist a strictly compac subset $k$ of $\Omega$, a bounded subset $L$ of $E, \varepsilon>0$ and $\alpha \in \mathbf{N}$ with $T \in V(K, L, \varepsilon, \alpha)^{\circ}$.

Let $\varphi$ be the function defined by

$$
\begin{aligned}
& \mathbf{R} \rightarrow \mathbf{R} \\
& u \mapsto \varphi(u)
\end{aligned}= \begin{cases}2 e \exp \left(1 /\left(u^{2}-1\right)\right) & \text { if }|u|<1 \\
0 & \text { if }|u| \geqslant 1\end{cases}
$$

$\varphi \in \mathscr{D}(\mathbf{R})$ and there is a constant $C \geqslant 0$ such that

$$
\sup _{\substack{u \in \mathbf{R} \\ k=0 \cdots \alpha}}\left|\varphi^{(k)}(u)\right| \leqslant C .
$$

For every $n \in \mathbf{N}^{*}$ and $x \in E$ we set $f_{n}(x)=a_{n}^{n} \varphi\left[2\left(a_{n}^{-1} v_{n} \circ s_{V}(x)-1\right)\right]$; thus $f_{n} \in$ $\mathcal{E}(E)$ and for $k \leqslant \alpha$

$$
\left|f_{n}^{(k)}(x) h_{1} \cdots h_{k}\right| \leqslant a_{n}^{n} C 2^{k} a_{n}^{-k}\left|v_{n} \circ s_{V}\left(h_{1}\right)\right| \cdots\left|v_{n} \circ s_{V}\left(h_{k}\right)\right| .
$$

Since $B$ is a bounded set, $\sup _{h \in L}\left|v_{n} \circ s_{V}(h)\right| \leqslant b$ for some constant $b$. But since $\left(2^{k} b^{k} C a_{n}^{n-k}\right)_{n \in \mathrm{N}^{*}}$ converges to zero, there is $N_{0} \in \mathrm{N}^{*}$ such that $f_{n} \leqslant V(K, L, \varepsilon, \alpha)$ if $n \geqslant N_{0}$.

From the following equivalence:

$$
f_{n}(x) \neq 0 \Leftrightarrow \frac{1}{2} a_{n}<v_{n} \circ s_{V}(x)<\frac{3}{2} a_{n}
$$

and

$$
P(x)=0 \Leftrightarrow v_{n} \circ s_{V}(x)=0 \quad \forall n \in \mathbf{N}^{*},
$$

$f_{n}$ is zero in a neighborhood of the zero set of $P$. Therefore $f_{n} / P$ makes sense and is an element of $\mathcal{E}(\Omega)$. If $N \geqslant \sup \left(n_{0}, N_{0}\right)$,

$$
\begin{aligned}
\left\langle T, f_{N}\right\rangle & =\left\langle T, P f_{N} / P\right\rangle=\left\langle S, f_{N} / P\right\rangle \\
& =\sum_{n \geqslant n_{0}} n^{-2}\left(f_{N} / P\right)\left(n^{-1} j_{n}\right) \geqslant N^{-2}\left(f_{N} / P\right)\left(N^{-1} j_{N}\right) .
\end{aligned}
$$

But $P\left(N^{-1} j_{N}\right)=a_{N}^{N} N^{-2}\left(v_{N}\left(e_{N}\right)\right)^{2}$ and $f_{N}\left(N^{-1} j_{N}\right)=2 a_{N}^{N}$. Therefore if $N \geqslant$ $\sup \left(n_{0}, N_{0}\right),\left|\left\langle T, f_{N}\right\rangle\right| \geqslant 2$. We get a contradiction.

Second part: If $S \in \mathcal{E}^{\prime}(\Omega)$, there is a strictly compact subset $K$ of $\Omega$, a bounded subset $B$ of $E$, an $\varepsilon>0$ and an $\alpha \in \mathbf{N}$ such that $S \in V(K, B, \varepsilon, \alpha)^{\circ}$. We may assume $K \subset B$ and $B$ convex balanced. Therefore $S_{\mid \Omega \cap E_{B}} \in \mathcal{E}^{\prime}\left(\Omega \cap E_{B}\right)$. We suppose that for every convex balanced 0 -neighborhood $V$ in $E$, $\operatorname{dim} s_{V}\left(E_{B}\right)<\infty$. Let $P$ be a nonzero continuous polynomial on $E$. The set $\Delta=\{x \in E /|P(x)|<|P(0)|+1\}$ is a neighborhood of zero; therefore there exists a convex balanced 0 -neighborhood $V$ such that $V \subset \Delta$. Let $E_{B}$ be endowed with the topology induced by that of $E$. Therefore $E_{B}=\left(E_{B} \cap \rho_{V}^{-1}(0)\right) \oplus F$ topologically with $F \simeq s_{V}\left(E_{B}\right)$. If $x \in E_{B} \cap V$, there are $u \in E_{B} \cap \rho_{V}^{-1}(0)$ and $v \in F$ such that $x=u+v$. If $\lambda \in \mathbf{R}$, we have $\lambda u+v=(\lambda-1) u+u+v$ and $\rho_{V}(\lambda u+v) \leqslant|\lambda-1| \rho_{V}(u)+\rho_{V}(u+v)<1$. Therefore, given $u \in E_{B} \cap \rho_{V}^{-1}(0)$ and $v \in F$, the polynomial $\lambda \mapsto P(\lambda u+v)$ is bounded on $\mathbf{R}$, and so a constant. Thus we have $P(u+v)=P(v)$ for all $u \in E_{B} \cap$ $\rho_{V}^{-1}(0)$ and so $P$ is a finite-type polynomial on $E_{B}$. According to Theorem 1 , there 
exists a $T_{1} \in \mathcal{E}^{\prime}\left(\Omega \cap E_{B}\right)$ such that $P_{\mid \Omega \cap E_{B}} T_{1}=S_{\mid \Omega \cap E_{B}}$. Let $r$ be the restriction map from $\mathcal{E}(\Omega)$ to $\mathcal{E}\left(\Omega \cap E_{B}\right)$ and ${ }^{t} r$ be its transpose. $T={ }^{t} r\left(T_{1}\right)$ is a solution of $P T=S$.

Let $\mathcal{G}$ be the class of the Hausdorff real locally convex spaces such that for every convex balanced 0 -neighborhood $V$, and every convex balanced bounded subset $B$ of $E$, we have $\operatorname{dim} s_{V}\left(E_{B}\right)<\infty$.

THEOREM 2'. Let $E$ be a Hausdorff real locally convex space and $\Omega$ an open subset of $E$. The division by any continuous analytic function which does not vanish on any open subset of $\Omega$ is always possible in $\mathcal{E}^{\prime}(\Omega)$ if and only if $E \in \mathcal{S}$.

Proof. We use the same proof as in Theorem 2, replacing polynomial by analytic function.

RemarKs. (1) Theorems 2 and $2^{\prime}$ are no more valid if we omit the continuous property. If $E \notin \mathcal{G}$, let us consider on $E$ the weak topology $(E, \sigma)$. Since $E$ and $(E, \sigma)$ have the same bounded sets, the topological spaces of the $C^{\infty}$ functions on $E$ and on $(E, \sigma)$ are also the same. The division by a polynomial $P$ in $(E, \sigma)$ is equivalent to the division in $E$. If $P$ is the polynomial of the counterexample, the division by $P$ is not possible in $(E, \sigma)$ and $(E, \sigma) \in \mathcal{G}$. We deduce from Theorem 2 that the polynomial $P$ is not continuous on $(E, \sigma)$.

(2) If $(E,\|\cdot\|)$ is a Banach space and if $E \in \mathcal{G}$, it is obvious that $E$ is finite dimensional. So:

Corollary 1. Let $E$ be a real Banach space, $E$ is a finite dimensional space if and only if the division of any distribution by any nonzero continuous polynomial is always possible in $\mathcal{E}^{\prime}(E)$.

(3) If $E$ is a Fréchet space and if $E \in \mathcal{G}$, we easily prove that, for every convex balanced 0-neighborhood $V, \operatorname{dim} s_{V}(E)$ is finite. The unique Fréchet spaces having this property are isomorphic to $\mathbf{R}^{n}$ or to $\mathbf{R}^{\mathbf{N}}$. (Let $E^{\prime}$ be the dual of $E$, equipped with the equicontinuous bornology; since $\operatorname{dim} s_{V}(E)<\infty,\left(E^{\prime}\right)_{V}^{0}$ is finite dimensional. If $\left(e_{i}^{\prime}\right)_{i \in I}$ is an algebraic basis of $E^{\prime}, E^{\prime}$ is bornologically isomorphic to $\mathbf{R}^{(I)}$. Let $E^{\prime x}$ be the bornological dual of $E^{\prime}$ equipped with the topology of the uniform convergence on the equicontinuous subsets of $E^{\prime}$. Therefore $E^{x} \simeq \mathbf{R}^{I}$ topologically. Since $E$ is a subspace of $E^{\prime x}$ and $E^{\prime x}$ is a Schwartz space, thus $E$ is a Schwartz space. Since $E$ is a complete Schwartz space, $E=E^{\prime x}$ (see Hogbe-Nlend [5, p. 95]).)

CoROllary 2. If $E$ is an infinite dimensional Fréchet space, $E \in \mathcal{G}$ if and only if $E \simeq \mathbf{R}^{\mathbf{N}}$.

(4) Let $F$ be a Fréchet space and $E$ its strong dual. If we suppose $E \in \mathcal{G}$, we prove that $F \in \mathcal{G}$ : if $B$ is a convex balanced bounded subset of $F$ and $V$ a convex balanced neighborhood of zero, then $\left(F_{B}, \rho_{V}\right)^{\prime} \simeq s_{B}^{0}\left(E_{V}^{0}\right)$. Since $E \in \mathcal{G}, \operatorname{dim} s_{B}^{0}\left(E_{V}^{0}\right)<\infty$ and $F_{B} / \rho_{V}^{-1}(0) \cap F_{B}$ is a finite dimensional space.

Since $F$ is a Fréchet space and $F \in \mathcal{G}$, according to (3), $F \simeq \mathbf{R}^{n}$ or $F \simeq \mathbf{R}^{\mathbf{N}}$. We deduce $E \simeq \mathbf{R}^{n}$ or $E \simeq \mathbf{R}^{(\mathbb{N})}$. 
COROllaRY 3. If $E$ is the strong dual of an infinite dimensional Fréchet space, $E \in \mathcal{G}$ if and only if $E \simeq \mathbf{R}^{(\mathbf{N})}$.

(5) If $E$ is a nuclear bornological vector space, the Fourier transform is a bornlogical and topological isomorphism between $\mathcal{E}^{\prime}(E)$ and $\mathscr{F}_{\mathcal{E}}(E)$ (see J. M. Ansemil and J. F. Colombeau [1]). Let $P$ be a nonzero real polynomial on $E$; we define the convolution operator $\theta$ by

$$
\text { 0: } \mathscr{F} \mathcal{E}^{\prime}(E) \rightarrow \mathscr{F E}^{\prime}(E), \quad \mathscr{F} f \mapsto \mathscr{F}(P f) .
$$

It is obvious that the surjectivity of the operator $\theta$ is equivalent to the surjectivity of the mapping

$$
\mathcal{E}^{\prime}(\Omega) \rightarrow \mathcal{E}^{\prime}(\Omega), \quad f \mapsto P f .
$$

According to Theorem 2, we deduce that for some $\mathcal{F}^{\prime}(E)$ all convolution operators are not surjective.

ACKNowledgements. The author is very grateful to J. F. Colombeau and B. Perrot for their help and to C. O. Kiselman for his encouragement.

\section{REFERENCES}

1. J. M. Ansemil and J. F. Colombeau, The Paley-Wiener-Schwartz isomorphism in nuclear spaces, Rev. Roumaine Math. Pures Appl. 26 (1981), 169-181.

2. J. F. Colombeau, Sur les applications différentiables et analytiques au sens de J. Sebastião e Silva, Portugal. Math. 33 (1977), 103-118.

3. On some various notions of infinite dimensional holomorphy, Proceedings on Infinite Dimensional Holomorphy, Lecture Notes in Math., Springer-Verlag, Berlin and New York, vol. 364, 1974, pp. 145-149.

4. J. F. Colombeau, R. Gay and B. Perrot, Division by holomorphic functions and convolution equations in infinite dimension, Trans. Amer. Math. Soc. 264 (1981), 381-391.

5. H. Hogbe-Nlend, Bornologies and functional analysis, Math. Studies, no. 26 North-Holland, Amsterdam, 1977.

6. L. Hörmander, On the division of distributions by polynomials, Ark. Mat. 3 (1958), 555-568.

7. S. Lojasiewicz, Sur le problème de la division, Studia Math. 18 (1959), 87-136.

8. L. Schwartz, Division par une fonction holomorphe sur une variété analytique complexe, Summa Brasil. Math. 3 (1955), 181-209.

U. E. R. DE Mathematiques et InformatiQue, Universite de Bordeaux I, 33405 Talence, France 